Ann. Zootech., I980, $29(4), 365-376$.

\title{
Régulation thermique de l'agneau nouveau-né : analyse de quelques facteurs influençant l'évolution de la température interne et cutanée de l'agneau nouveau-né
}

\author{
A. DUSSUEL $\left({ }^{(1)}\right.$, A. KOBILINSKY $\left({ }^{2}\right)$ et P. BERBIGIER $\left({ }^{(}\right)\left({ }^{*}\right)$ \\ (1) Station Centrale de Bioclimatologie. \\ (2) Station de Biométrie, I.N.R.A., Versailles. \\ 78000 Versailles (France)
}

\begin{abstract}
Résumé
On a étudié l'influence de certaines caractéristiques d'agneaux nouveau-nés (poids, portée, race, type de toison,,.. ) et des conditions de température et d'humidité ambiante sur l'évolution des températures rectales et cutanées (dos) pendant les 18 premières heures de la vie. L'expérience a eu lieu en bergerie non chauffée et a porté sur 85 animaux. Les mesures de température ont été effectuées o h, I h, $2 \mathrm{~h}, 4 \mathrm{~h}, 6 \mathrm{~h}, \mathrm{I} 2 \mathrm{~h}$ et $\mathrm{I} 8 \mathrm{~h}$ après la naissance. La température cutanée, très variable, $\left(30^{\circ}\right.$ à $\left.3^{\circ}\right)$ présente des oscillations d'amplitude importante. La température rectale est plus élevée et beaucoup moins variable, son évolution montre que l'animal réagit đans les quelques heures après la naissance pour réguler sa température interne. Cette dernière présente d'abord des oscillations de plusieurs dixièmes de degré, puis se stabilise entre $6 \mathrm{~h}$ et $12 \mathrm{~h}$ après la naissance. Les agneaux d'une même portée évoluent de façon semblable. Sont sans doute à l'origine de ce fait les facteurs nutritionnels dont l'intensité varie avec la fréquence des tétées, la quantité et qualité du lait ingéré, d'où l'importance des relations mère-enfant au début đe la vie đe l'agneau. On montre également que la température rectale :

- est peu influencée par les facteurs climatiques dans ces conditions expérimentales;

- augmente avec le poids de l'animal.
\end{abstract}

\section{I. - Introduction}

La mortalité chez les agneaux est importante. Elle s'observe surtout pendant la première semaine où elle représente plus de $5^{\circ} \mathrm{p}$. Ioo des pertes totales (LEFEBVRE, I976). On enregistre des taux de mortalité moyens d'environ 77 p. roo (Purser \& Young, I959; Gunn et Robinson, I963), mais pouvant atteindre

(*) Adresse actuelle : I.N.R.A., Antilles, Domaine Duclos, 97 I 70 Petit-Bourg (Guadeloupe). 
25 p. Ioo (Houston \& Maddox, I974) et même parfois 45 p. Ioo de l'effectif des animaux nés (GunN, cité par Sykes, Griffiths \& SLEE, I976). Les causes de mortalité sont nombreuses, mais le plus souvent, elle intervient après un épuisement des réserves énergétiques (Houston et MaDDox, 1974) qui ne sont renouvelées que si le jeune s'alimente sans retard après la naissance. Or ALEXANDER et WIILIAMs (rg66) ont montré que l'activité de recherche de tétée décroît avec 1'hypothermie. Par conséquent, l'établissement rapide de la thermorégulation après la naissance est important pour la survie de l'agneau.

L'étude présente a pour objectif de décrire et modéliser l'évolution des températures corporelles pendant les 18 premières heures de vie de 1'agneau et de dégager les facteurs intervenant de façon prépondérante sur cette évolution. L,es facteurs pris en compte sont d'une part des mesures de température et d'humidité dans 1'atmosphère ambiante, d'autre part des caractéristiques facilement observables de l'animal. On a ainsi retenu celles qui peuvent influencer la thermorégulation, soit au travers de phénomènes physiologiques, soit par un effet physique direct, soit encore par leur action sur l'intensité et la qualité des rapports entre la mère et le jeune.

\section{II. - Matériel et méthodes}

\section{I. - Animaux et mesures}

L'expérience a eu lieu dans une bergerie non chauffée (température ambiante variant entre 7 et I $^{\circ}{ }^{\circ} \mathrm{C}$ ) au Centre de Recherches de Clermont-Ferrand. Elle a porté sur 85 agneaux appartenant à cinq types génétiques différents : 36 Romanov $\times$ Limousin $(R \times L), 35$ Ile-de-France $\times$ (Romanov $\times$ Limousin) $(I \times$ $(\mathrm{R} \times \mathrm{L})), 9$ Ile-de-France $\times$ Ile-de-France $(\mathrm{I} \times \mathrm{I}), 3$ Romanov $\times$ Ile-de-France $(\mathrm{R} \times \mathrm{I})$ et 2 Ile-de-France $\times$ Limousin $(\mathrm{I} \times \mathrm{L})(\mathrm{La}$ race du père est indiquée en premier).

Compte tenu des contraintes expérimentales du troupeau et du nombre de facteurs pouvant avoir une éventuelle action sur la régulation de température, il n'était pas possible de réaliser un véritable plan d'expérience. On a donc pris l'ensemble des animaux nés pendant la période de l'expérience (I semaine), sans les soumettre à des traitements climatiques particuliers. Pour chaque agneau nouveau-né, on a mesuré la température de surface (TS) de la peau du dos (zone interscapulaire) avec un thermomètre à thermistance, et la température rectale (ou interne TI) avec un thermomètre médical. En même temps, on a déterminé la température (TA) et 1'humidité de l'air (température du point de rosée : TR) avec un psychromètre placé au-dessus de 1'animal. Ces mesures ont été effectuées à o h (en fait 5 à I $5 \mathrm{mn}$ après la naissance suivant 1'animal), puis I h, $2 \mathrm{~h}, 4 \mathrm{~h}, 6 \mathrm{~h}$, $\mathrm{I} 2 \mathrm{~h}$, et $\mathrm{I} 8 \mathrm{~h}$ après $1 \mathrm{a}$ mise-bas. On a vérifié que la température radiative moyenne des parois de la bergerie, était toujours égale (à $\pm{ }^{\circ} \mathrm{C}$ près) à la température de l'air, et que la vitesse de l'air dans la bergerie était faible.

$\left(<0,2 \mathrm{~ms}^{-1}\right)$.

On a enfin noté pour chaque agneau les niveaux de certains facteurs liés au jeune ou à sa mère susceptibles d'influencer l'évolution des températures corporelles, à savoir: la race de l'agneau, le sexe de l'agneau, le type de toison (on a distingué 3 types; poils bouclés et courts, poils longs et lisses, poils bouclés et longs. Le type de toison est lié à la race : les agneaux $I \times I, R \times I, I \times L$, ont des poils bouclés 
et courts, les agneaux $\mathrm{R} \times \mathrm{L}$ des poils longs et lisses; seuls les agneaux $\mathrm{I} \times(\mathrm{R}$ $\times$ L) présentent les 3 types de toison, et celui-ci n'a pu être déterminé pour $I 7$ d'entre eux), le poids à la naissance, la taille de portée : I, 2, 3 ou 4 agneaux, la parité de la mère (on a distingué mère primipare, multipare d'âge inférieur à 7 ans et multipare d'âge supérieur ou égal à 7 ans en fonction de données sur la mortalité des agneaux suivant 1'âge et la parité de la mère (Mac DonaL, I966)), le numéro de la mère (facteur " effet de la mère "), la prise de sang : en effet dans le cadre d'une expérience parallèle à la nôtre, des prises de sang étaient effectuées sur certains animaux à la naissance et avant nos mesures de températures.

\section{2. - Remarque sur la mesure de la température de surface (TS)}

Les mesures de températures corporelles étaient faites par deux opérateurs A et B, A travaillant le jour et B la nuit. Or on a constaté que les mesures de température de surface faites par $A$ étaient systématiquement plus élevées d'environ $\mathrm{I}^{0}$ que les mesures faites par B. On a alors corrigé les mesures de A en leur retranchant la différence entre les moyennes des mesures effectuées par A d'une part, par $B$ de l'autre, ceci à chaque temps de mesure (o h, I h, 2 h. ...).

\section{3. - Traitement statistique}

Il permet de préciser l'effet des facteurs pris en compte sur l'évolution des températures corporelles pendant les i 8 premières heures de vie de l'agneau.

La méthodologie retenue pour l'analyse statistique est identique à celle qui est décrite par BERBIGIER et al. (I978). Etant donné le grand nombre de facteurs et le faible effectif d'animaux concernés, on n'a pas retenu d'interaction. Le modéle additif initial choisi pour expliquer la température de surface ou rectale interne au temps $t\left(\mathrm{TS}_{t}, \mathrm{TI}_{t}\right)$ comprend donc :

- un terme pour chacun des facteurs qualitatifs décrit ci-dessus;

- un terme pour chacune des covariables : température de l'air et du point de rosée, poids à la naissance;

- un terme pour chacune des covariables : température de surface ou rectale aux temps précédents.

Dans le cadre de ce modèle, on a effectué les tests ajustés de chacun des facteurs et réduit le modèle par une procédure qui s'apparente à la régression progressive descendante.

Le test d'un facteur est ajusté pour l'ensemble des autres facteurs figurant dans le modèle global précédemment décrit. En particulier, l'ajustement pour les températures corporelles aux temps précédents (dans le cas où $t \geqslant I \mathrm{~h}$ ) implique qu'on étudie l'évolution de la température au cours du temps. Pour faciliter l'interprétation des tests ajustés et le choix des rotations des termes du modèle dans l'étape de réduction, on a analysé d'abord les liaisons entre facteurs et covariables. Deux analyses ont été effectuées à cette fin. La première est une analyse en Composantes Principales sur les températures de l'air et đu point de rosée (TA, TR) aux différents temps de mesure. La seconde, décrite par KobILrNsky (I980) a permis d'étudier les liaisons entre les différents facteurs qualificatifs - excepté le facteur mère - ainsi que les liaisons entre ces facteurs et le poids.

L'effet de la mère n'a pas été introduit au départ de l'analyse, car nous pensions qu'il se réduisait à l'effet de la température rectale initiale et des autres fac- 
teurs liés à la mère. Par la suite, nous avons constaté que cette hypothèse n'était que partiellement vraie. Nous avons alors réintroduit l'effet mère dans le modèle et procédé à une nouvelle analyse de variance avec récupération de l'information inter mère.

\section{III. - Résultats}

\section{1. - Liaisons entre les différents facteurs}

I) L'analyse en composantes principales sur les températures externes fait apparaître une première valeur propre nettement supérieure aux autres. L'axe correspondant est corrélé positivement à l'ensemble des températures, avec des corrélations voisines de $+0,9$. L'interprétation est simple : la fluctuation de la température de l'air et du point de rosée sur 18 h est faible quand on la compare aux différences entre les températures observées tout au long de l'expérience.

Dans l'interprétation des analyses qui suivent, on négligera la variation des températures de l'air (TA) et du point de rosée (TR) sur les I 8 heures pendant lesquelles chaque agneau est observé. L'effet d'une quelconque des covariables " $T A_{0}, \ldots, T_{18}, T R_{0}, \ldots, T_{18}$ " sera interprêté comme l'effet de la température moyenne pendant les 18 premières heures de vie de l'animal.

2) L'analyse des liaisons entre facteurs qualitatifs montre que l'échantillon recueilli permet surtout d'apprécier la différence d'effet entre les 2 races les plus représentées $\mathrm{R} \times \mathrm{L}, \mathrm{I} \times(\mathrm{R} \times \mathrm{L})$, sans toutefois pouvoir dissocier l'effet toison, les différences d'effet entre tailles de portées (surtout entre les tailles de portées 2 et 3) la différence entre mère primipare et non primipare, et enfin l'effet sexe.

Par ailleurs, si l'effet du sexe peut être étudié indépendamment des facteurs " mère " et " poids ", il n'en est pas de même des effets de la race, du numéro de portée, de la taille de portée, qui sont indissociables de l'effet mère. Ils ont en outre (notamment le dernier) une influence non négligeable sur le poids.

Enfin, l'examen des degrés de liberté dans l'analyse intramère a montré que les seules associations de types de toison rencontrées dans une même portée (race $\mathrm{I} \times(\mathrm{R} \times \mathrm{L})$ sont : bouclé long, bouclé court d'une part, lisse et long, non noté d'autre part. Le contraste " poil lisse — poil bouclé " ne peut donc être dissocié de l'effet mère.

Étant donné les fortes liaisons entre facteurs, et le nombre de résultats déduits des tests, on a retenu seulement dans l'analyse de variance les faits les plus nets et les plus réguliers. C'est pourquoi, les effets à la limite de la signification et apparaissant de façon épisodique au cours du temps n'ont pas été retenus dans l'interprétation des résultats (les tests correspondants sont suivis d'un point d'interrogation dans les tabl. 2 et 3 ).

\section{2. - Variabilité des températures de surface et rectale}

La variabilité résiduelle de la température de surface (TS) est nettement supérieure à celle de la température interne (TI). TS présente en outre des oscillations importantes entre o et 18 heures, de l'ordre de plusieurs degrés, qui ne sont liées ni aux fluctuations de TI ni aux facteurs pris en compte. C'est donc l'étude de TI qui donne les résultats les plus significatifs. 
TABLEAU I

Liaisons entre températures corporelles aux différents temps Relations between body temperatures at different times
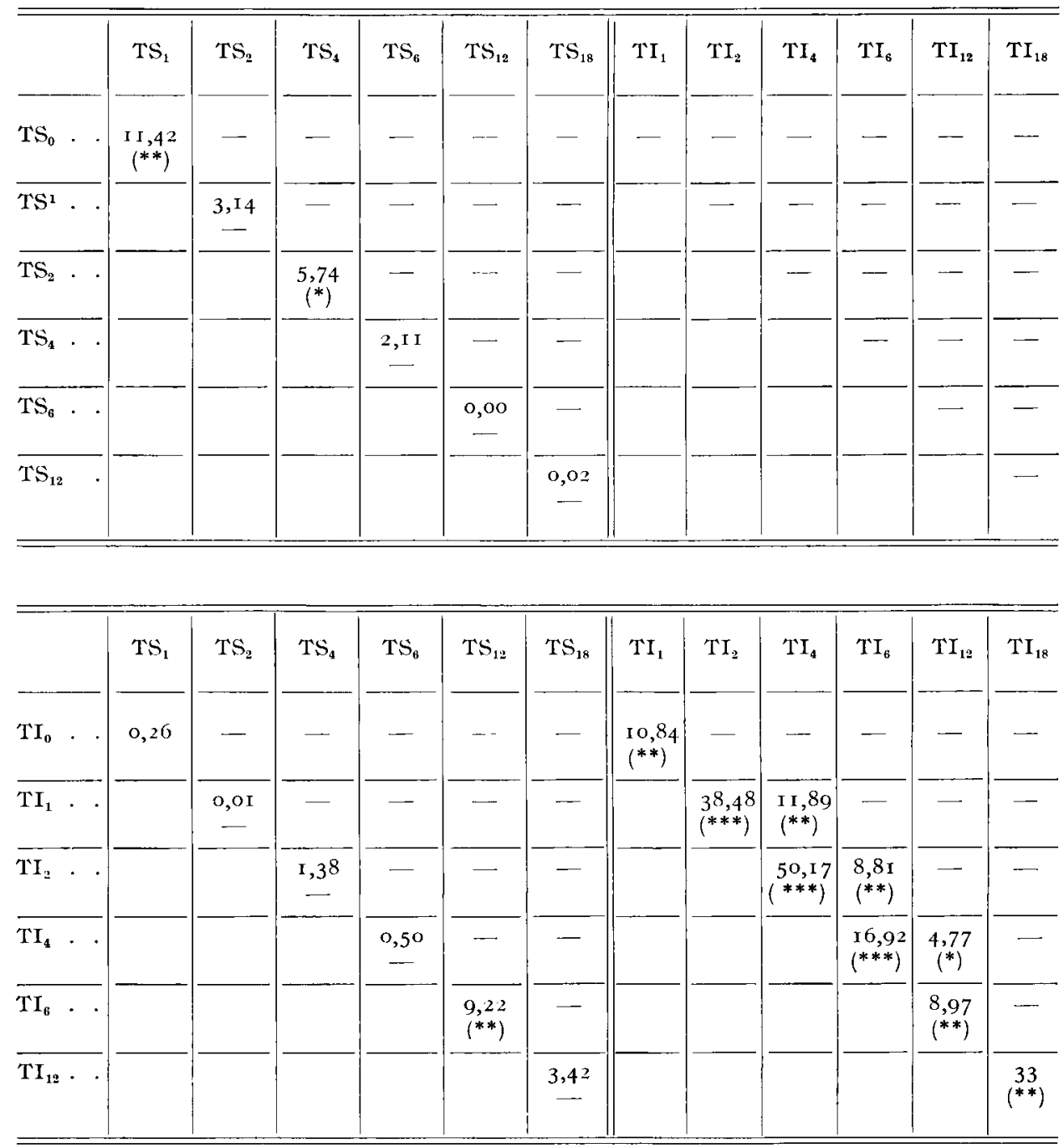

Note : - TS $=$ température de surface. $T I=$ température interne ou rectale.

-- I a variable expliquée est en colonne - la ligne indique la covariable testée.

- I,es nombres indiquent la valeur du $\mathrm{F}$ calculé (test $\mathrm{F}$ ).

... Signification $\left(^{*}\right)=5$ p. $100 ;\left(^{*}\right)=$ I p. I00 $;\left(^{* *}\right)=$ I p. 1000 .

$-T S=S k i n$ temperature $-T I=$ Rectal temperature.

- Explained variable are in column, tested covariables in row.

- Numbers give the value of $F$ - tests.

- Significance: $\left({ }^{*}\right)=5$ p. Ioo, $\left({ }^{* *}\right)=I$ p. I0o; $(* * *)=I$ p. Iooo. 
3.3. - Effets des températures de l'air et de point de rosée (TR) sur les cinétiques de températures de surface $(T S)$ et rectale $(T I)$

Bien que le domaine des températures ambiantes soit assez grand (TA variant entre 7 et $\mathrm{I}^{\circ} \mathrm{C}$ ), on ne trouve pas d'effet très significatif et répété.

\section{4. - Liaisons entre températures corporelles (TS et TI)}

La température de surface (TS) au temps $t_{i}$ est peu liée à la température de surface ou interne au temps précédent $t_{i-1}$. Par contre, il existe une forte dépendance pour la température interne ('TI) : 'TI au temps $t_{i}$ est liée à TI au temps précédent $t_{i-1}$ et même parfois à TI au temps $t_{i_{-2}}$ avec des seuils de signification élevée (I p. Ioo ou I p. IOOO), mais la température interne (TI) n'est jamais liée à la température de surface (TS) au temps précédent (tabl. $x$ ).

Une analyse en composantes principales des valeurs de la température interne confirmie se résultat. On trouve trois sous-groupes : $\mathrm{TI}_{0}$ et $\mathrm{TI}_{1}$ qui sont fortement

\section{TABLEAU 2}

Effets des facteurs liés au jeune et à sa mère sur la température de surface (TS)

Effects of factors depending on the lamb and on the dam for surface temperature (TS)

\begin{tabular}{|c|c|c|c|c|c|c|c|}
\hline Facteurs (Factors) & $\mathrm{TS}_{0}$ & $\mathrm{TS}$ & $\mathrm{TS}_{2}$ & $\mathrm{TS}_{4}$ & $\mathrm{TS}_{6}$ & $\mathrm{TS}_{12}$ & $\mathrm{TS}_{18}$ \\
\hline $\begin{array}{l}\text { Mère primip. ou multip. (pri- } \\
\text { miparous or multiparous } \\
\text { ewe) } . \cdot \cdot \cdot \cdot \cdot \cdot \cdot \cdot .\end{array}$ & - & - & - & - & & $\begin{array}{c}3,77 \\
(*)(?)\end{array}$ & 一 \\
\hline Taille de portée (litter size) & - & - & - & - & 一 & - & - \\
\hline Prise de sang (blood sampling) & $\begin{array}{c}6,32 \\
(*)\end{array}$ & - & - & - & - & $\begin{array}{c}4,93 \\
(*) \text { (?) }\end{array}$ & - \\
\hline 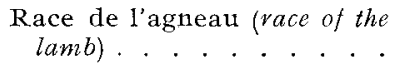 & $\begin{array}{r}4,34 \\
(* *)\end{array}$ & $\begin{array}{l}4,32 \\
(* *)\end{array}$ & 一 & - & 一 & -- & $\begin{array}{c}2,57 \\
(*)(?)\end{array}$ \\
\hline Type de toison (fleece). . . & — & $\begin{array}{l}4, \text { II } \\
(*) \text { (?) }\end{array}$ & $\ldots$ & - & - & - & - \\
\hline $\begin{array}{c}\text { Poids à la naissance (birth } \\
\text { weight) . . . . . . . . . }\end{array}$ & $\begin{array}{l}19,72 \\
(* * *)\end{array}$ & - & - & - & - & - & - \\
\hline Sexe de l'agneau (lamb's sex) & - & $(* *), 64$ & - & - & - & - & - \\
\hline
\end{tabular}

Note : Les nombres indiquent la valeur du $\mathrm{F}$ calculé (test $\mathrm{F}$ ).

- Signification : * $=5$ p. xoo, $(* *)=I$ p. I000, $(* * *)=$ I p. Iooo.

- (?) : Effet difficile à interpréter, parce qu'il est isolé et peu significatif.

Numbers give the value of $F$ test.

- Significance: $(*)=5$ p. xoo, $(* *)=x$ p. Iooo, $(* * *)=\mathbf{I} p$. I000.

- (?) Isolated, scarcely significant effect, not clearly explained. 
liées, $\mathrm{TI}_{2}$, $\mathrm{TI}_{4}$ et $T \mathrm{TI}_{6}$ pour lesquelles la liaison est plus faible et enfin $\mathrm{TI}_{12}$ et $\mathrm{TI}_{18}$ qui sont très fortement liées entre elles. Vraisemblablement, si ces groupes existent, c'est parce que, au cours de la première heure l'agneau n'a pas encore bu de lait alors qu'après $\mathbf{2} 2$ heures il a ingéré du lait et sa thermorégulation est active.

\section{5. - Effets des facteurs liés au jeune et à sa mère}

\subsection{1. - Effet des facteurs autre que la mère.}

La parité de la mère, la taille de la portée et le sexe de l'agneau n'influencent pas de façon significative la température de surface (TS) et rectale (TI). Les facteurs agissant de façon prépondérante sont la prise de sang, la race ou le type de toison de l'agneau et surtout le poids à la naissance. L'influence du poids sur TS se manifeste de façon très significative (I \%) seulement après la naissance (5 à I5 mn après la mise-bas) (tab1. 2).

Quant à la température rectale, elle est liée au poids de o à $\mathrm{I} 8$ heures de vie et principalement pendant la première heure (significatif à I \%). Pour préciser l'effet du poids sur la température rectale, on a formé des classes de poids, puis on a porté sur un graphique les moyennes de température rectale par classe de poids en fonction du temps (fig. I). Les animaux de la classe de poids supérieure à $4,5 \mathrm{~kg}$ ont toujours une température rectale plus haute que les autres, les classes intermédiaires ( $\mathrm{P}$ compris entre 2,5 et $4,5 \mathrm{~kg}$ ) ont des températures à peu près semblables. I'un des agneaux de poids très faible $(P=I, 6 \mathrm{~kg})$ a une température rectale nettement inférieure à celle des autres animaux à la naissance; elle ne fait que

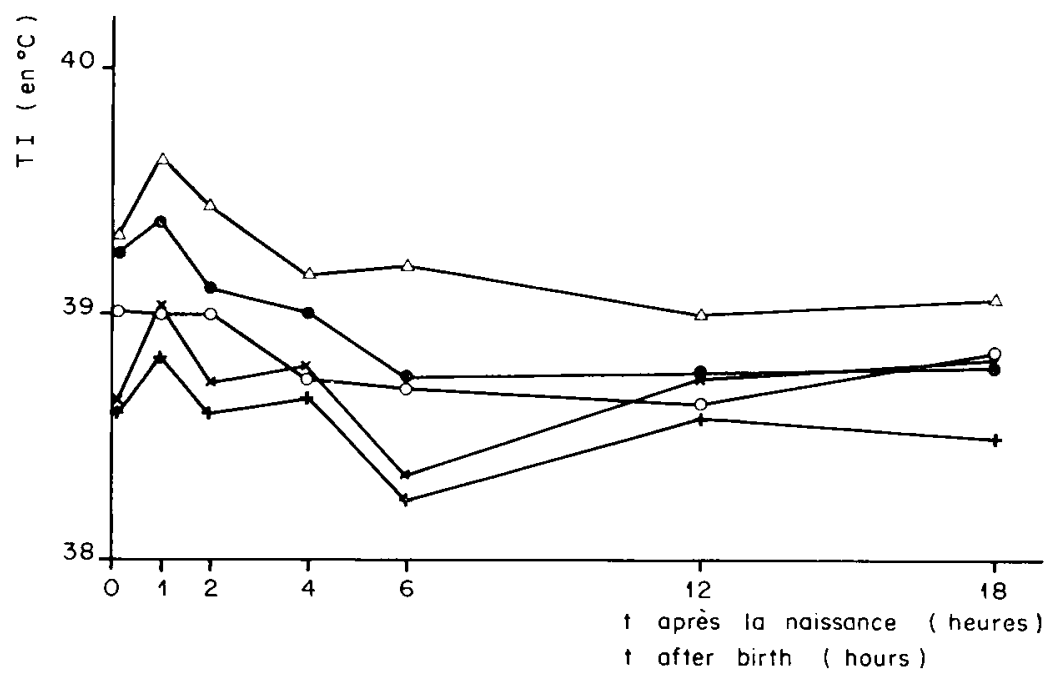

FIG. I. - Cinétiques des moyennes de température rectale par classe de poids. Kinetics of mean vectal temperatures for each birth'weight class

$\triangle \mathrm{P}>4,5 \mathrm{~kg}$

- $3,5<P \leqslant 4,5 \mathrm{~kg}$

- $2,5<P \leqslant 3,5 \mathrm{~kg}$

$+\mathrm{P} \leqslant 2,5 \mathrm{~kg}$

$\times P \leqslant 2,5 \mathrm{~kg}$ moins l'agneau $\mathrm{P}=1,6 \mathrm{~kg}$ 
décroitre par la suite ( $\mathrm{TI}=35^{\circ}$ à I $8 \mathrm{~h}$ ). Si l'on ne prend pas en compte cet agneau qui n'a pas survécu, la température moyenne des animaux de plus faible poids $(\mathrm{P} \leqslant 2,5 \mathrm{~kg})$ est inférieure jusqu'à $\mathrm{I} 2$ heures et sensiblement égale ensuite à celle des classes de poids intermédiaires.

Comme nous l'avons signalé auparavant, cette première analyse ne tient pas compte de l'effet mère. Aussi, les effets observés pour les différents facteurs, tous plus ou moins liés à la mère (sauf le sexe), peuvent-ils être attribués à une différence entre mères. Dans la seconde analyse, qui prend en compte l'effet maternel, seul le poids a gardé un effet significatif. Le faible nombre de mères observées (30) n'a donc pas permis de dissocier de l'effet mère, les effets des autres facteurs.

\subsection{2. - Effet de la mère}

Les tests de l'effet mère sur les températures internes à I heure, 2 heures,... après la naissance sont reportés dans le tableau 3 .Ces tests sont ajustés pour l'ensemble des facteurs connus à o heure, y compris $\mathrm{TI}_{\mathbf{0}}$. On remarque des effets signi-

TABLEAU 3

Effet des facteurs liés au jeune et à sa mève sur la températuve vectale (TI) Effects of factors depending on the lamb and on the dam for rectal temperature

\begin{tabular}{|c|c|c|c|c|c|c|c|}
\hline Facteurs (Factors) & $\mathrm{TI}_{0}$ & $\mathrm{TI}_{1}$ & $\mathrm{TI}_{2}$ & $\mathrm{TI}_{4}$ & $\mathrm{TI}_{6}$ & $\mathrm{TI}_{12}$ & $\mathrm{TI}_{18}$ \\
\hline $\begin{array}{l}\text { Mère primi-ou multipare ( } p r i- \\
\text { mi or multiparous erve) }\end{array}$ & - & - & $(*)(?)$ & - & - & - & 一 \\
\hline Taille de portée (litter size) & - & - & - & - & - & - & - \\
\hline Prise de sang (blood sampling) & $\begin{array}{c}4,98 \\
(*)\end{array}$ & $\stackrel{4,37}{(*)}$ & $\begin{array}{c}5,47 \\
(*)\end{array}$ & $\begin{array}{c}3,95 \\
(*)\end{array}$ & - & - & - \\
\hline $\begin{array}{l}\text { Race de l'agneau (breed of the } \\
\operatorname{lamb}(\cdot \ldots \cdot \ldots\end{array}$ & - & - & - & - & $\begin{array}{l}3,05 \\
(*)(?)\end{array}$ & - & - \\
\hline Type de toison (fleece) $\ldots$ & - & $\begin{array}{l}8,55 \\
(* * *)\end{array}$ & $\begin{array}{c}3,89 \\
(*)\end{array}$ & $\begin{array}{l}4,25 \\
(* *)\end{array}$ & 一 & - & - \\
\hline $\begin{array}{c}\text { Poids à la naissance (bivth } \\
\text { qeeight) } \\
.\end{array}$ & $\begin{array}{l}13,72 \\
(* * *)\end{array}$ & $\begin{array}{r}19,04 \\
(* * *)\end{array}$ & $\begin{array}{l}4,64 \\
(*)\end{array}$ & $\begin{array}{r}4,02 \\
\left({ }^{*}\right)\end{array}$ & $\begin{array}{l}13,83 \\
(* * *)\end{array}$ & $\begin{array}{c}6,07 \\
(*)\end{array}$ & $\begin{array}{c}\text { IO, I } 4 \\
(*)\end{array}$ \\
\hline Sexe de l'agneau (sex). & - & - & - & - & - & - & - \\
\hline Effet mère (maternal effect) & & $\begin{array}{c}2, \mathrm{II} \\
(*)\end{array}$ & & $\begin{array}{l}2,72 \\
(* *)\end{array}$ & $\begin{array}{l}3,73 \\
(* * *)\end{array}$ & & \\
\hline
\end{tabular}

Légende : Cf. tableau 2.

L'effet mère est ajusté pour tous les facteurs figurant dans le tableau ainsi que pour la température rectale initiale (TI ). En revanche, les autres effets ne sont pas ajustés pour l'effet mère.

Matermal effect is adjusted for every other factor listed in the table and for rectal initial temperature. Other effects are of course not adjusted for maternal effect. 
ficatifs, notamment à 4 heures et 6 heures après la naissance : donc l'évolution de TI entre o et 6 heures est fortement influencée par la mère.

Pour illustrer l'effet mère, nous avons tracé sur une même figure les cinétiques de température rectale de tous les agneaux d'une portée, ainsi que la moyenne de température rectale des agneaux de cette même portée ceci pour une trentaine de portées. La figure 2 donne les courbes obtenues pour quatre portées. Ce sont 4 profils types. La comparaison des figures obtenues pour chaque portée montre que les températures rectales des agneaux d'une même mère suivent à peu près le même profil, et que la variabilité est plus grande entre mères qu'entre agneaux d'une même mère, du moins pendant les 6 ou $\mathrm{r} 2$ premières heures de vie, où la température rectale fait de nombreuses oscillations de nature très différentes suivant la mère. A partir de 6 ou I 2 heures, la température se stabilise à un niveau à peu près constant compris entre $38^{\circ} \mathrm{C}$ et $39,5^{\circ} \mathrm{C}$, quelle que soit la portée.

De plus en général, si la température rectale initiale $\left(\mathrm{TI}_{0}\right)$ est supérieure à $39^{\circ} \mathrm{C}$ ou $39,5{ }^{\circ} \mathrm{C}$, on observe une chute de la température rectale pendant la première heure (fig. 2, portées 2 et 3 ); par contre si $T I_{0}$ est inférieure à 39 ou $39,5^{\circ} \mathrm{C}$, on a une augmentation de la température rectale pendant la première heure (fig. 2 , portées $n^{\circ}$ I, 3 et 4 ).
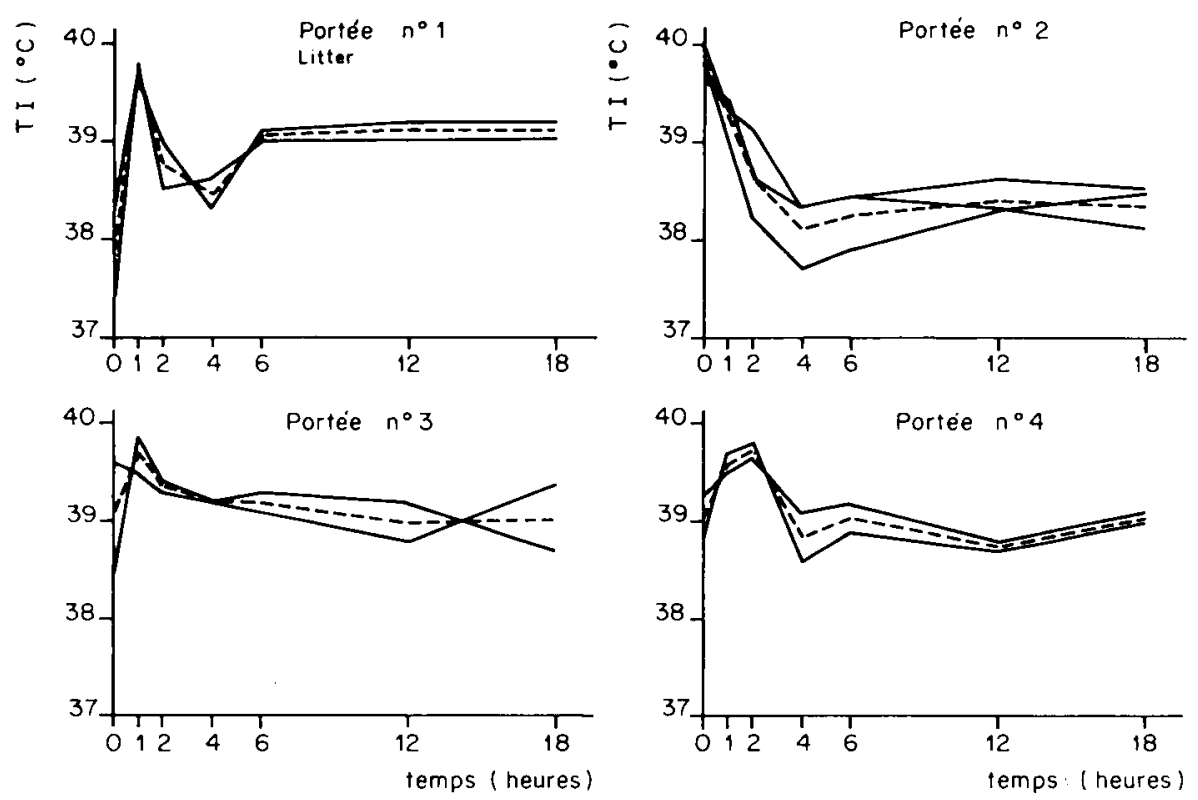

FIG. 2. - Cinétiques de température rectale des agneaux nouveau-nés par portée

Kinetics of rectal temperature of new-born lambs according to the litter un agneau de la portée. (one lamb in the litter).

...- moyenne des $n$ agneaux de la portée (mean temperature in the litter).

\section{6. - Modèles minima prévisionnels pour les températures corporelles (TS et $T I$ )}

La qualité des modèles sélectionnées à l'aide de la méthode de " réduction " peut être représentée par les pourcentages de la variance totale expliquée par le modèle. Ces pourcentages étant constamment inférieurs à $20 \mathrm{p}$. roo pour la tem- 
pérature de surface, nous donnerons seulement leurs valeurs et les modèles associés pour la température rectale (TI).

(5 p. Iоo) $\mathrm{TI}_{0}=39$, IO $+0,22(\mathrm{P}-3,4)$.

$\left(26\right.$ p. IOo) $\mathrm{TI}_{1}=39, \mathrm{I} 7+0,28(\mathrm{P}-3,4)+0,3 \mathrm{I}\left(\mathrm{TI}_{0}-39,0\right)$.

(48 p. Iоo) $\mathrm{TI}_{2}=38,89+0,60\left(\mathrm{TI}_{1}-39,0\right)$.

(56 p. гоo) $\mathrm{TI}_{4}=38,77+0,29\left(\mathrm{TI}_{2}-39,0\right)+0,3 \mathrm{I}\left(\mathrm{TI}_{1}-39,0\right)+0,04\left(\mathrm{TA}_{4}-\mathrm{I}_{3}, 0\right)$.

(52 p. roo) $\mathrm{TI}_{6}=38,77+0,47\left(\mathrm{TI}_{4}-39,0\right)+0,2 \mathrm{I}\left(\mathrm{TI}_{2}-39,0\right)+{ }_{2}^{\prime} \mathrm{o,I} 2(\mathrm{P}-3,4)$.

(27 p. Iо0) $\mathrm{TI}_{12}=38,82+0,28\left(\mathrm{TI}_{6}-39,0\right)+0,26\left(\mathrm{TI}_{4}-39,0\right)$

(4I p. Iоo) $\left(\mathrm{TI}_{18}=39,04+0,76\left(\mathrm{TI}_{12}-39,0\right)\right.$.

Ainsi la variation de $\mathrm{I} \mathrm{kg}$ de poids corporel provoque une variation de TI de $0,22{ }^{\circ} \mathrm{C}$ à o heure, mais seulement de $0,12{ }^{\circ} \mathrm{C}$ à 6 heures, et la température rectale à un instant donné dépend essentiellement des températures rectales aux instants précédents.

La température de surface TS dépend essentiellement de la température de surface ou rectale à l'instant précédent.

\section{IV. - Discussion et conclusion}

\section{I. - Sur la variabilité des mesures suivant l'observateur}

Les différences constatées entre les valeurs de la température de surface (TS) mesurées par les deux observateurs méritent d'être discutées. L'un des observateurs appuyait plus que l'autre la pointe métallique du thermomètre à thermistance sur la surface de peau, entraînant une perturbation du flux sanguin superficiel, ce qui peut expliquer une valeur différente de la température de surface. Par ailleurs, l'un des observateurs effectuait ses mesures systématiquement le jour, l'autre travaillait toujours la nuit.

La première explication nous semble la plus plausible, puisqu'on a observé une très faible différence de température ambiante (TA) et d'humidité ambiante (TR) sur I8 heures de mesure pour un même animal.

On a constaté aussi que la température de surface (TS) présentait des oscillations d'amplitude importante. Ceci pose le problème de la fiabilité et de la précision de la mesure, effectuée sur une surface de 2 à $3 \mathrm{~mm}$ de diamètre et qui peut varier à l'intérieur de la zone interscapulaire du dos de l'animal.

Par ailleurs, il faudrait mesurer la température de surface (TS) sur différentes parties du corps pour savoir si elle est uniforme ou non. Ce type d'étude serait nécessaire pour obtenir un bilan des échanges de chaleur global par la méthode du bilan d'énergie (BERBIGIER, I975). A partir des résultats partiels obtenus par AI.EXANDER (I 96I), on observe en effet que la température de surface au niveau du pied est beaucoup plus basse que celle du tronc. Aussi, il nous paraît plus intéressant de retenir les valeurs de la température rectale (TI) pour l'interprétation des résultats.

\section{2. - Evolution et variation de la température interne}

Les facteurs climatiques (TA et TR) ont peu d'influence, du moins dans les conditions climatiques de notre expérience. Ceci rejoint des résultats antérieurs de Griffiths (I968) observés pour des températures ambiantes comprises entre I5 
et $35^{\circ} \mathrm{C}$, et d'AlEXANDER (I96I) qui trouvait seulement un effet à la limite de la signification pour $\mathrm{TA}$ compris entre 5 et $40^{\circ}$. Le maintien, généralement jugé nécessaire, d'une température de 15 à $20^{\circ}$ en bergerie d'agnelage, n'est donc nullement indispensable à l'établissement d'une bonne régulation thermique de l'agneau nouveau-né. Ėn fait, d'après ALEXANDER, l'agneau nouveau-né peut supporter des températures inférieures à $0^{\circ} \mathrm{C}$ en l'absence de vent.

I a taille de portée et la parité de la mère n'ont plus d'influence, une fois pris en compte le poids à la naissance de l'agneau. Le sexe n'a pas d'influence non plus. Par contre, on observe les influences du type de toison pendant les 4 premières heures, de la mère pendant les 6 premières heures et surtout du poids, pendant les I 8 premières heures de mesure. Ceci confirme les résultats d'autres auteurs (Sy KEs, GrIfFiths et Sl,EE I 976, GRIfFIThS, I968). Il n'a cependant pas été possible de dissocier l'effet du type de toison de l'effet maternel à partir de nos données.

L'étude de l'évolution des courbes individuelles de température rectale (TI) (fig. 2), ainsi que les fortes liaisons entre températures rectales aux différents temps montrent que l'animal réagit rapidement pour réguler sa température interne : dès la première heure, donc avant ingestion de lait et alors que l'animal est encore mouillé, on assiste à une augmentation de TI si cette dernière a chuté assez fortement pendant les premières minutes après la naissance. Ceci avait déjà été mis en évidence par (Alexander et MCCANCE (I958) et Griffitiss (I968). Il $\mathrm{y}$ a donc augmentation de la production de chaleur ou diminution des pertes thermiques grâce à l'établissement de phénomènes de vaso-constriction. Douze heures après la naissance, la température est à peu près stabilisée : l'agneau a alors bu du lait, et sa thermorégulation est active. Ce développement rapide de l'homéothermie avait déjà été mentionné par AlExander (I96I) \& GRIFriths (I968).

Il est impossible d'analyser davantage les oscillations qui précèdent la stabilisation sans avoir fait parallèlement aux mesures décrites précédemment des notations concernant les apports alimentaires (horaires des tétées, quantité et qualité du lait ingéré).

Les modèles prévisionnels pour TI expliquent au plus $5^{\circ} \mathrm{p}$. I oo de la variance totale. En particulier, la variation de ' $\mathrm{II}_{0}$ n'est pratiquement pas expliquée. II faut done rechercher d'autres facteurs pouvant agir sur l'évolution de la température interne. On peut penser à des facteurs complémentaires d'ordre métabolique comme la maturité physiologique, la température interne de la mère, ou encore aux apports nutritionnels susceptibles d'expliquer les fluctuations similaires des températures des agneaux d'une même portée.

Cette étude n'était qu'une expérience préliminaire pour rechercher les principales causes de variation des températures corporelles chez l'agneau nouveau-né. Elle mériterait d'être poursuivie en utilisant des plans expérimentaux plus rigoureux utilisant les résultats de cette première expérience. Elle pourrait être enfin utilement complétée par des mesures ponctuelles du métabolisme énergétique de l'agneau nouveau-né.

Accepté pour publication en octobre 1980.

\section{Remerciements}

Au Laboratoire de la Production Ovine (C. R. Z. V. 'THEIx) pour nous avoir fourni le matériel animal utilisé dans cette étude. 


\section{Summary \\ Temperature regulation in the new-born lamb Factors affecting rectal and skin surface temperatures}

A study was made in new-born lambs to examine the in fluence of some parameters (weight, litter, breed, fleece,...) and of room temperature $\left(7-18^{\circ} \mathrm{C}\right)$ and relative humidity on rectal and skin surface (back) temperatures during the first 18 hours of life. The experiment, involving 85 animals, was conducted id an unheated sheep-fold. Body temperatures were measured $o, I$, $2,4,6,12$ and 18 hours after birth. Highly variable $\left(3 \circ\right.$ to $\left.38^{\circ}\right)$, the skin surface temperature showed very large oscillations, which could not be explained by the other factors studied. Rectal temperature was higher and less variable $\left(38-40^{\circ}\right)$. Its variations (fig. 2) showed that the animal is regulating its internal temperature within the first hours following birth. After several oscillations, the rectal temperature became stable between 6 and $I 2$ hours after birth. Lambs from the same litter exhibited nearly the same temperature variations which could be explained by nutritional factors such as sucking frequency as well as quantity and quality of ingested milk.

Under these experimental conditions, there was no relationship between room temperature, humidity and body temperature, thus suggesting that it is not necessary to keep a temperature above $15^{\circ}$ in lambing pens, as generally admitted.

On the basis of the present data, effects of other factors cannot be dissociated from the large maternal effect, except for weight. Figure $x$ shows that rectal temperature of heavy lambs remained higher during the 18 hours of the experiment.

\section{Références bibliographiques}

Alexander G., I96I. Temperature regulation in the new-born lamb. III. - Effect of environmental temperature on metabolic rate, body temperatures and respiratory quotient. Austr. J. Agric. Res., 12, II 52-II 74 .

AlEXANDER G., MC CANCE I., I958. Temperature regulation in the new-born lamb. I. C Changes in rectal temperature within the first six hours of life. Austr. J. Agric. Res., 9, 339-347.

AlEXANDER G., WILliams D., I966. Teat seeking activity in new-born lamb: the effect of cold. J. Agric. Sci. Camb., 67, I8I-189.

BERBigier P., r975. Sur la mesure des échanges de chaleur au niveau de la peau des porcs élevés sous abri par la méthode du bilan d'énergie. Ann. Zootech., 24, 413-422.

Berbigier P., Le Dividich J., Kobilinsky A., I 978 . Échanges thermiques chez le porcelet nouveau-né : application de la méthode du bilan d'énergie. Ann. Zootech., 27, I8I-I94.

GRIFFITHS J. G., I968. Observations on neonatal changes of body temperature in Blackface lambs. Anim. Prod., 10, 3r9-323.

GunN R. G., Robinson J. F., I963. Lamb. mortality in Scottish hill flocks. Anim. Prod., 5, $67-76$.

Houston D. C., Madmox J. G., I974. Causes of mortality among young Scottish Blackface lambs. Vet. Rec., 95, 575.

KoBILINsky A., I980. Décomposition de formes quadratiques en analyse de données. Thèse $3^{\mathrm{e}}$ cycle Univ. Paris Sud. Orsay. Annexe, I-I4.

LEFEBVRE, D., I976. Aspects quantitatifs de la mortalité des agneaux. Application au cas des agneaux de race Lacaune, Sarde, FSL allaités artificiellement. Mémoire de fin d'études, E.N.S.A. Montpellier, $77 \mathrm{p}$.

Mac Donal, J. W., I 966 . Variation in perinatal mortality of lambs with age and parity of ewes. Proc. Austr. Soc. Anim. Prod., 6, 60-62.

Purser A. F., Young G. B., I959. Lamb survival in two hill flocks. Anim. Prod., 1, 85-9I.

Sykes A. R., GRIfitis R. G., SLEE J., 1976. Influence of breed, birth weight and weather on the body temperature of new-born lambs. Anim. Prod., 22, 395-402. 